\title{
Infraspinatus scapular retraction test: a reliable and practical method to assess infraspinatus strength in overhead athletes with scapular dyskinesis
}

\author{
Giovanni Merolla Elisa De Santis · Fabrizio Campi • \\ Paolo Paladini · Giuseppe Porcellini
}

Received: 30 January 2010/ Accepted: 7 May 2010/Published online: 1 June 2010

(c) The Author(s) 2010. This article is published with open access at Springerlink.com

\begin{abstract}
Background Alteration of normal scapulohumeral rhythm due to the fatigue of scapular-stabilizing muscles induces decrease of rotator cuff strength. In this study we analyzed the interobserver and intraobserver realibility of the infraspinatus strength test (IST) and infraspinatus scapular retraction test (ISRT) in 29 overhead athletes with scapular dykinesis, before and after 6 months of scapular musculature rehabilitation.

Materials and methods Subjects with magnetic resonance imaging (MRI) findings of labral injuries (2 cases, $5 \%$ ) and cuff tears (4 cases, 11\%) were excluded. Scapular dyskinesis patterns were evaluated according to Kibler et al. (J Shoulder Elbow Surg 11:550-556, 2002). We found a type I dyskinesis in 24 cases (83\%) and a type II in 5 cases (17\%). Patients were tested by using IST and ISRT and the maximum infraspinatus strength $(\mathrm{kg})$ was registered by a handheld dynamometer. Changes in shoulder IR were measured by using a standard goniometry. Rehabilitation continued for 6 months and was focused on the restoration of scapular muscular control and balance. We used a paired Student $t$ test for the significance of the force values $($ alpha $=0.01)$. Intraclass correlation coefficient $($ ICC) and standard error (SE) were applied to determine the realibility of repeated values collected within testers and between testers.
\end{abstract}

G. Merolla $(\bowtie)$ - F. Campi · P. Paladini · G. Porcellini

Unit of Shoulder and Elbow Surgery, "D. Cervesi" Hospital, L.V Beethowen 46, 47841 Cattolica, RN, Italy

e-mail: gmerolla@shouldertech.it

E. De Santis

ShoulderTech Clinical and Rehabilitative Office, Forlì, Italy
Results Values of ICC close to 1 at baseline and at 6 months indicated a higher interexaminer and intraexaminer realibility. IST force values registered a significant increase at 6 months for both examiners $(P<0.01)$. The mean difference between IST and ISRT values were not significant at 6 months $(P>0.01)$. The increase of glenohumeral internal rotation was significant at 6 months $(P<0.01)$.

Conclusion The good realibility and the easy reproducibility make the ISRT an excellent test to assess patients with infraspinatus weakness due to scapular dyskinesis and address them toward an appropriate program of rehabilitation aimed to restore scapular musculature balance and control.

Keywords Infraspinatus - Strength - Scapular dyskinesis . Realibility

\section{Introduction}

Overhead translation of the glenohumeral joint requires a balance activity of scapular-stabilizing muscle to maintain the so-called scapulohumeral rhythm, that is, the gliding motion of the anterior surface of the scapula on the posterolateral surface of the thoracic cage [1]. The fatigue of the scapular stabilizer muscles alters scapulohumeral rhythm and induces a decrease of the rotator cuff (RC) strength. Kibler et al. [2] explored the relationship between apparent supraspinatus weakness and scapular dyskinesis, while Smith et al. [3] reported the effect of the scapula repositioning on isometric shoulder elevation strength. In our previous paper [4] we described a practical test to assess infraspinatus strength with the scapula retracted (infraspinatus scapula retraction test, ISRT) versus 
infraspinatus strength with the scapula free (infraspinatus strength test, IST) in professional volleyball players with scapular dyskinesis. In the current study we analyzed the interobserver and intraobserver realibility of IST and ISRT in 29 overhead athletes with altered scapular kinesis, before and after 6 months of rehabilitation.

\section{Materials and methods}

\section{Study population}

Thirty-five overhead athletes (21 volleyball players, 8 tennis players; age $23 \pm 4.5$ years; sex 16 male, 13 female; dominant arm 26 right, 3 left; height $178 \pm 4.6 \mathrm{~cm}$; weight $73 \pm 7.2 \mathrm{~kg}$; playing experience $7 \pm 1.8$ years) complaining of shoulder pain and weakness during their sports activity were selected for this study. Magnetic resonance imaging (MRI) was performed in all subjects; 2 cases with superior labral injuries $(5 \%)$ and 4 cases $(11 \%)$ with cuff tears were excluded. The final cohort enrolled included 29 subjects with an intact RC. All the patients gave informed consent prior to being included in the study. As this study was a standard of care, local ethics committee authorization was not required. The study was performed in accordance with the ethical standards of the 1964 Declaration of Helsinky as revised in 2000.

\section{Testing procedure}

Scapular dyskinesis patterns were evaluated according to Kibler et al. [5]. We found a type I dyskinesis (Fig. 1) in 24 cases $(83 \%)$ and a type II (Fig. 2) in 5 cases (17\%). The test was performed on the patient standing with the arm adducted, shoulder in neutral rotation, and elbow flexed at $90^{\circ}$. The first part of the test evaluated infraspinatus strength (IST) according to the criteria previously described in the literature [6, 7] (Fig. 3). The second part included a test administered with the scapula retracted by the examiner (ISRT) (Fig. 4). The maximum infraspinatus strength $(\mathrm{kg})$ after a voluntary isometric contraction was registered by a handheld dynamometer (Lafayette Instruments, Lafayette, Ind) (Fig. 5). Changes in isolated IR $\left({ }^{\circ}\right.$ ) of the humeral head on the glenoid were measured by using a standard goniometry [8] (Fig. 6).

\section{Rehabilitation program}

The rehabilitation program continued for 6 months and was focused on the restoration of scapular muscular control and balance [9] to obtain the following balance ratios: upper trapezius/lower trapezius (UT/LT) (Fig. 7), upper trapezius/middle trapezius (UT/MT) (Fig. 8), and upper

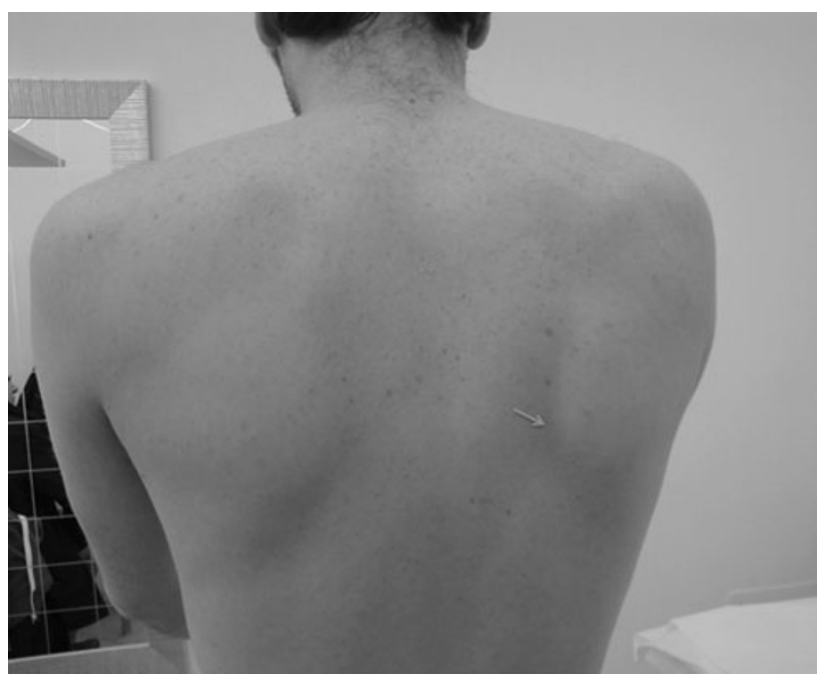

Fig. 1 Type I scapular dyskinesis

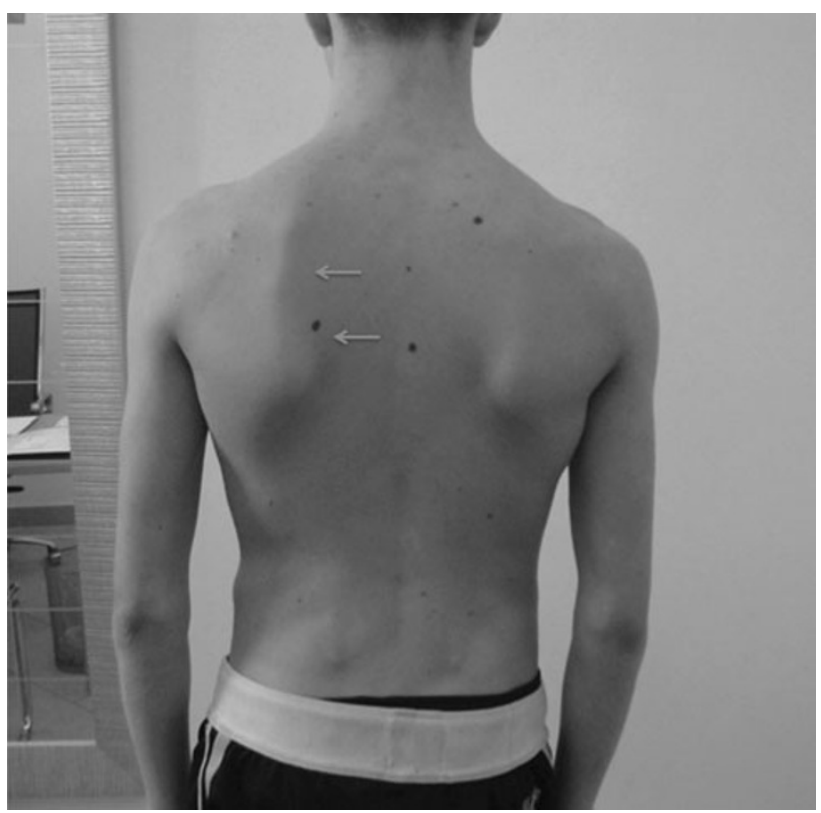

Fig. 2 Type II scapular dyskinesis

trapezius/serratus anterior (UT/SA) (Fig. 9). Active exercises for MT were associated with reinforcement of rhomboid muscles. Open kinetic chain exercises for shoulder girdle and posterior soft tissues stretching [10,11] were added to recover internal rotation deficit.

\section{Intraexaminer and interexaminer reliability}

Intraexaminer realibility was assessed in 3 testing sessions by two blinded examiners (GM and GP) on the patients randomly enrolled with $2 \mathrm{~h}$ between sessions. Examiner 1 recorded the maximum infraspinatus force $(\mathrm{kg})$ of 15 shoulder on 3 different evaluations, examiner 2 recorded 


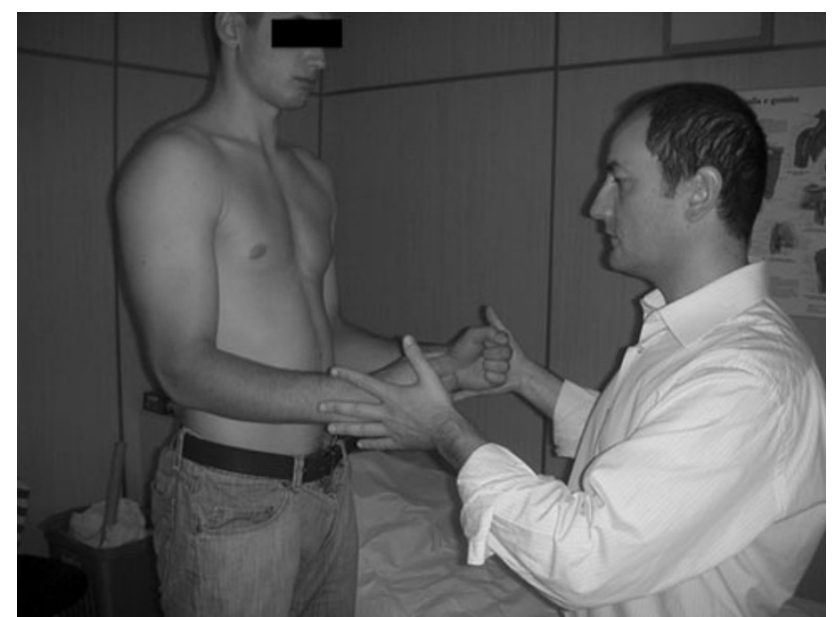

Fig. 3 Position of the patient and examiner for infraspinatus strength test (IST)

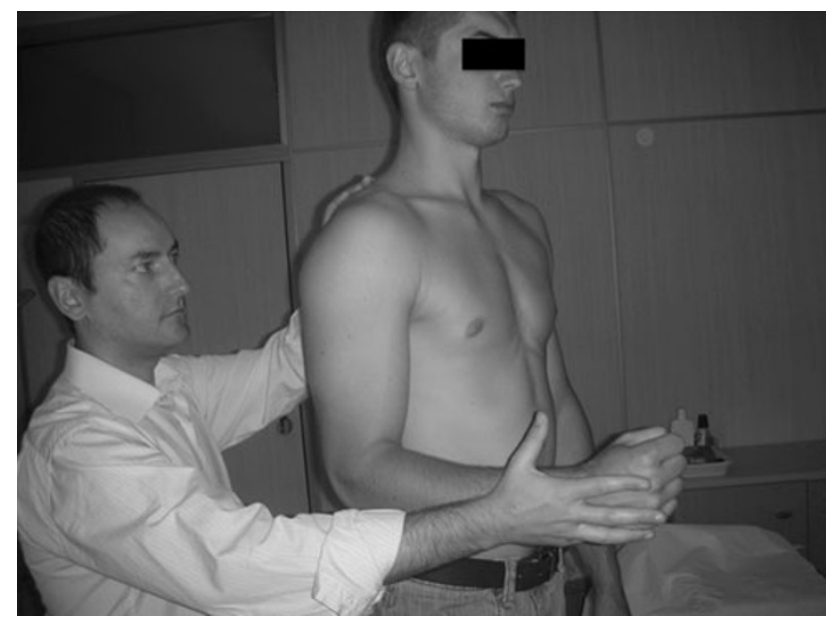

Fig. 4 Position of the patient and examiner for infraspinatus scapular retraction test (ISRT)

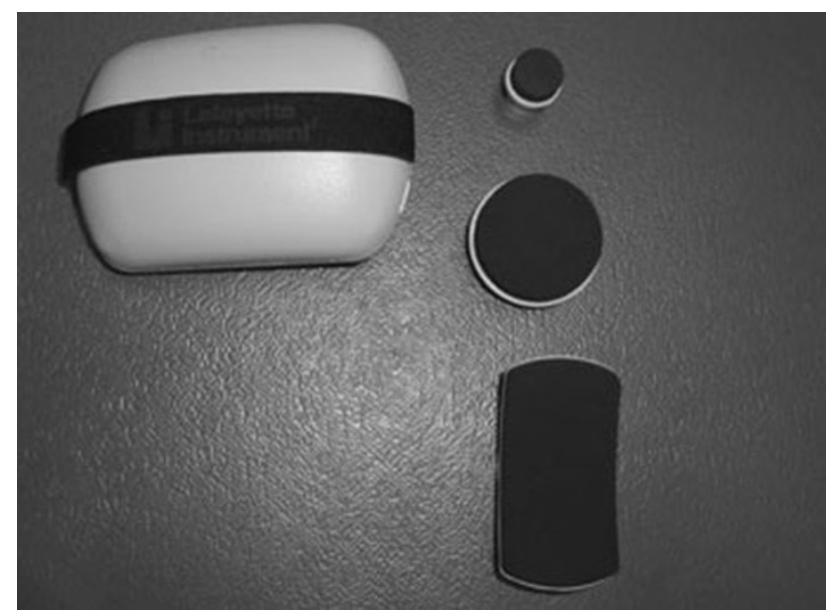

Fig. 5 Handheld dynamometer used to register infraspinatus strength

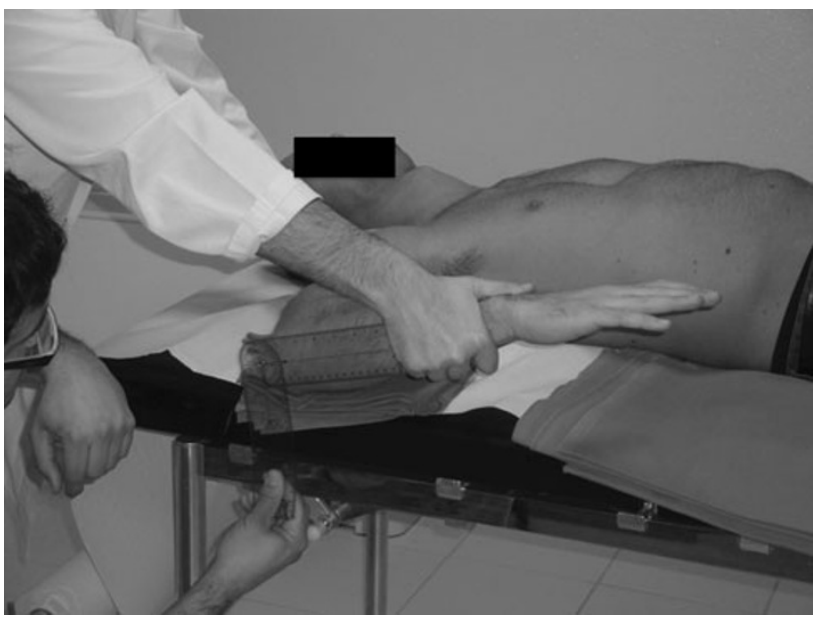

Fig. 6 Measurement of isolated glenohumeral internal rotation (GHIR)

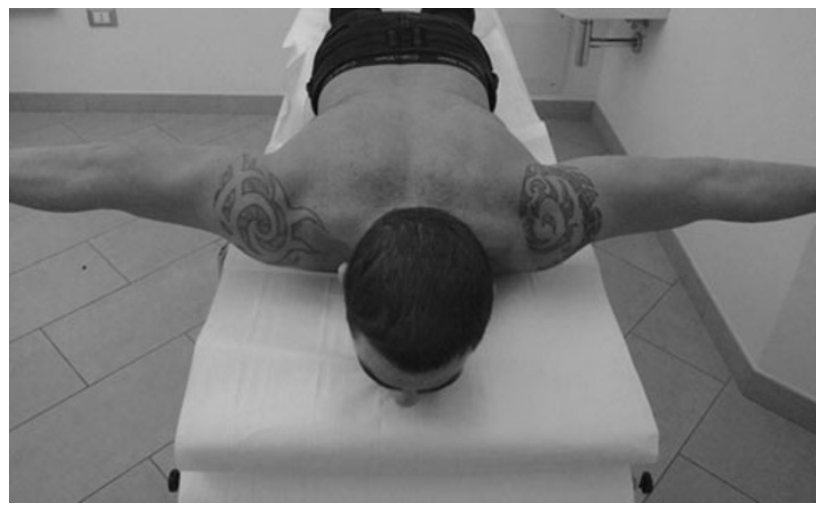

Fig. 7 Exercise to restore upper trapezius/lower trapezius (UT)/(LT) muscle balance

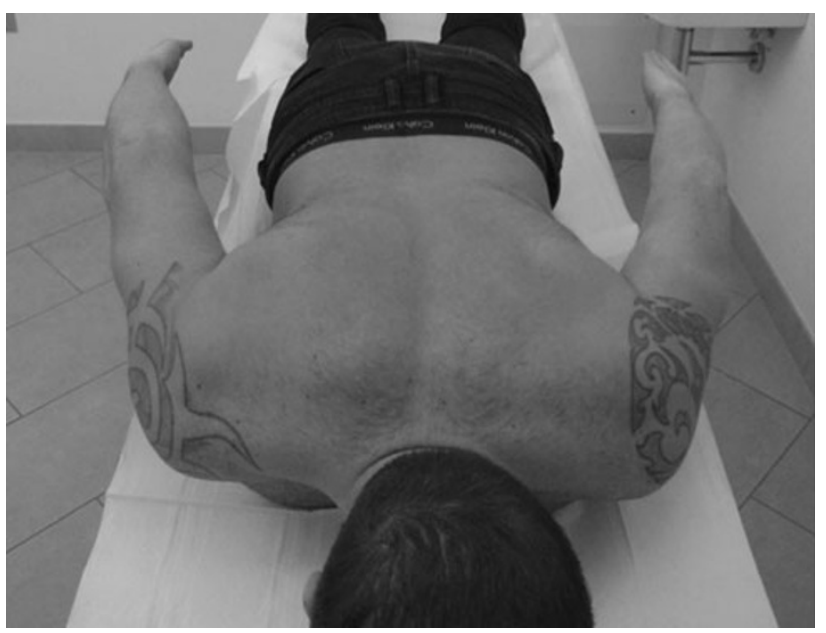

Fig. 8 Exercises to restore upper trapezius/middle trapezius (UT)/ (MT) muscle balance 


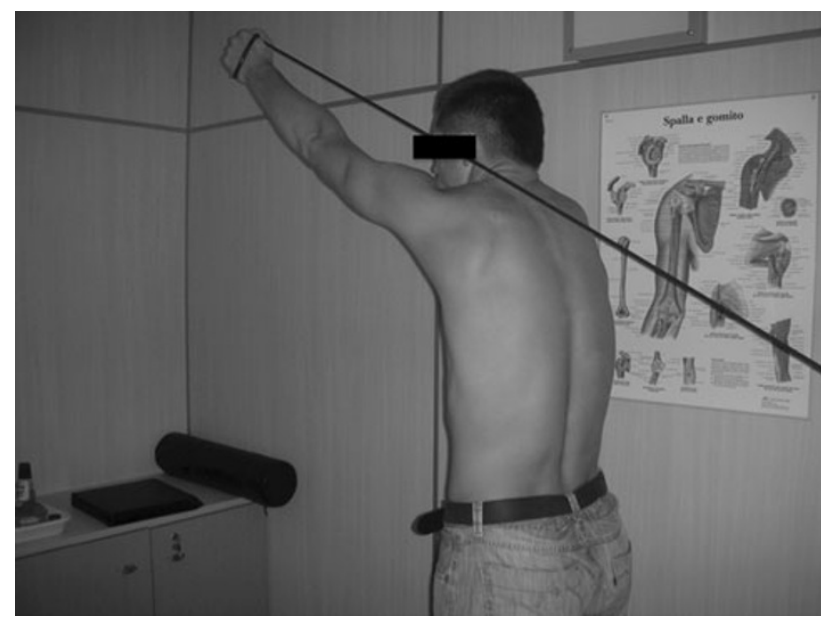

Fig. 9 Exercise to reinforce serratus anterior (SA) muscle

the other 14 shoulder on 3 different evaluations. Shoulder internal rotation was measured by using the same criteria of randomization and blindness. Examiners were both certified and expert orthopedic surgeons.

\section{Data analysis}

Strength values of the infraspinatus were registered at 3- and 6-month follow-up. We used a paired Student $t$ test for the significance of the force values [12] that was set at $1 \%$ (alpha $=0.01$ ). Intraclass correlation coefficient $($ ICC) and standard error (SE) were applied to determine the realibility of repeated values collected within testers and between testers.

\section{Results}

Results of the interexaminer realibility analysis of IST and ISRT before and after 6 months of rehabilitation are reported in Tables 1 and 2. Intraexaminer realibility resulted in ICC values between 3 sessions of 0.92 at baseline and 0.93 at 6 months. IST force values at 6 months registered a mean increase of $3.3 \pm 1.54$ for the examiner $1(P=0.0069)$ and $3.9 \pm 1.6$ for the examiner $2(P=0.0058)$. The mean difference between IST and ISRT values were not significant at 6 months $(P=0.061)$. Glenohumeral internal rotation (GHIR) increased at 6 months from $54.5 \pm 9.8$ to $67.3 \pm 10.1$ for the examiner $1 \quad(P=0.0096)$ (Fig. 6) and from $53.9 \pm 10.2$ to $68.1 \pm 11.4$ for the examiner $2(P=0.0089)$.

\section{Discussion}

The contribution of the scapula position for normal shoulder function has been widely described [3, 5, 13-17].
Table 1 Interexaminer realibility analysis of IST and ISRT force values $(\mathrm{kg})$ at baseline

\begin{tabular}{|c|c|c|c|}
\hline & \multicolumn{3}{|l|}{ Baseline } \\
\hline & IST $($ mean \pm SD) & SE & ICC \\
\hline Examiner 1 & $11.6 \pm 1.42$ & 0.39 & 0.91 \\
\hline \multirow[t]{3}{*}{ Examiner 2} & $11.3 \pm 1.61$ & 0.45 & \\
\hline & \multicolumn{3}{|l|}{ Baseline } \\
\hline & ISRT $($ mean \pm SD) & SE & $\mathrm{ICC}$ \\
\hline Examiner 1 & $16.1 \pm 2.1$ & 0.59 & 0.93 \\
\hline Examiner 2 & $15.9 \pm 2.8$ & 0.78 & \\
\hline
\end{tabular}

IST infraspinatus strength test, $S E$ standard error, ICC intraclass correlation coefficient, ISRT infraspinatus scapular retraction test

Table 2 Interexaminer realibility analysis of IST and ISRT after 6 months of rehabilitation

\begin{tabular}{llll}
\hline & \multicolumn{2}{l}{6 months } & \\
\cline { 2 - 4 } & IST (mean $\pm \mathrm{SD})$ & SE & ICC \\
\hline Examiner 1 & $14.9 \pm 2.6$ & 0.72 & 0.91 \\
Examiner 2 & $15.2 \pm 3.6$ & 1.01 & \\
\hline & 6 months & & \\
\cline { 2 - 4 } & ISRT (mean $\pm \mathrm{SD})$ & SE & ICC \\
\hline Examiner 1 & $15.4 \pm 2.1$ & 0.54 & 0.93 \\
Examiner 2 & $15.5 \pm 2.3$ & 0.59 & \\
\end{tabular}

$I S T$ infraspinatus strength test, $S E$ standard error, ICC intraclass correlation coefficient, ISRT infraspinatus scapular retraction test

Alteration in scapular muscle coordination and recruitment increases the demand on the RC musculature and metabolic cost of reaching forward elevation and secondarily results in RC weakness [18]. The inability of the scapula to provide a stable base of support for $\mathrm{RC}$ muscles produces a detrimental effect on isometric shoulder rotation strength [16] and an apparent supraspinatus weakness [2]. These research outcomes emphasize the role of the scapula in normal shoulder function that can be summarized in three points [19, 20]: (1) maintenance of dynamic stability and control glenohumeral joint mobility, (2) scapula as basis for muscle attachment, (3) scapula as the link in the proximal-to-distal transfer of energy for the appropriate shoulder positioning. Alterations in these functions play a part in shoulder dysfunction manifested with scapular protraction in patients with scapular dyskinesis (loss of acromial upward rotation, excessive scapular internal rotation, and excessive scapular anterior tilt) that results in decrease of the subacromial space with consequent decrease in $\mathrm{RC}$ strength and alteration of $\mathrm{RC}$ activation sequencing patterns [16]. About the specific contribution of 
the muscles attaching to the scapula, the LT and SA are effective in stabilizing the scapula during arm movement. A fatigue of these muscles disrupts the normal kinematics leading to the symptoms of impingement ("inhibition due to pain") [4]. These findings suggest that extrinsic (1) and intrinsic (2) factors are implicated in infraspinatus weakness: (1) abnormal scapular position due to the fatigue of scapular stabilizers creates an unstable base of support for RC muscles, (2) inhibition due to pain and disuse atrophy of muscle belly induce an apparent decrease of infraspinatus strength. Deficit of GHIR is a common finding associated with supraspinatus and infraspinatus dysfunction and can be explained as follows: (a) acceleration, deceleration, and follow-through phases of striking require $\mathrm{RC}$ muscles to act eccentrically to compress the humeral head [21]; (b) during spiking and serving, supraspinatus, infraspinatus, and teres minor muscles eccentrically resist translation and assist in deceleration of the moving limb [21, 22]; (c) the increased stress placed on the passive stabilizer of the shoulder [21, 22] leads to a posterior shoulder soft tissue tightness similar to that experience by throwing athletes [13]. For these reasons, posterior soft tissues stretching was included in the rehabilitation program [4] to act on posterior structural changes and minimize the effects on GHIR. Since the relationship between infraspinatus weakness and scapular disorders was not described in the literature, in our previous paper [4] we reported the experience collected with ISRT in the examination of overhead athletes with scapular disorders. Although that study showed the increase of IST force values was consistent with the recovery of scapular muscular balance, a realibility study of IST versus ISRT was not performed. Therefore, the goal of the current paper has been to elucidate this topic in a further cohort of athletes with similar demographic and clinical features. When the two evaluations were compared we found a non-significant difference in IST and ISRT force values at baseline (IST 0.03; ISRT 0.02) and at 6 months (IST 0.02; ISRT 0.01). ICC close to 1 indicated a higher intertester and intratester correlation in the two separate examinations. The good realibility and the easy reproducibility make the ISRT an excellent test to assess patients with infraspinatus weakness due to scapular dyskinesis and address them toward an appropriate program of rehabilitation aimed to restore scapular musculature balance and control. Some weak points are noteworthy in this study: (1) there is a lack of data concerning the position of the scapula before and after the rehabilitation, (2) testing was carried out by only two examiners and this might be a limit for the study realibility, (3) analysis of the scapular-stabilizing muscle patterns activation sequences was not performed. The points (1) and (2) need to be examined more deeply and elucidated in further investigations, while topic (3) is beyond the scope of the current paper and has been well discussed in other interesting recent research articles [23, 24].

\section{Conflict of interest None.}

Open Access This article is distributed under the terms of the Creative Commons Attribution Noncommercial License which permits any noncommercial use, distribution, and reproduction in any medium, provided the original author(s) and source are credited.

\section{References}

1. Kibler WB (1991) Role of the scapula in the overhead throwing motion. Contemp Orthop 22:525-532

2. Kibler WB, Sciascia A, Dome D (2006) Evaluation of apparent and absolute supraspinatus strength in patients with shoulder injury using the scapula retraction test. Am J Sports Med 34:1643-1647

3. Smith J, Kotajarvi BR, Padgett DJ, Eischen JJ (2002) Effect of scapular protraction and retraction on isometric shoulder elevation strength. Arch Phys Med Rehabil 83:367-370

4. Merolla G, De Santis E, Sperling JW, Campi F, Paladini P, Porcellini G (2010) Infraspinatus strength assessment before and after scapular muscles rehabilitation in professional volleyball players with scapular dyskinesis. J Shoulder Elbow Surg. doi: 10.1016/jse.2010.01.022

5. Kibler WB, Uhl TL, Maddux JW, Brooks PV, Zeller B, McMullen J (2002) Qualitative clinical evaluation of scapular dysfunction: a reliability study. J Shoulder Elbow Surg 11:550-556

6. Hawkins RJ, Kennedy JC (1980) Impingement syndrome in athletes. Am J Sports Med 8:151-158

7. Leroux JL, Thomas E, Bonnel F, Blotman F (1995) Diagnostic value of clinical tests for shoulder impingement syndrome. Rev Rhum Engl Ed 62:423-428

8. Norkin CC, White DJ (1985) Introduction to goniometry. In: Measurement of joint motion: a guide to goniometry. FA Davis, Philadelphia, pp 1-7

9. Rubin BD, Kibler WB (2002) Fundamental principles of shoulder rehabilitation: conservative to postoperative management. Arthroscopy 18:29-39

10. Laudner KG, Sipes RC, Wilson JT (2008) The acute effects of sleeper stretches on shoulder range of motion. J Athl Train 43:359-363

11. Johansen RL, Callis M, Potts J, Shall LM (1995) A modified internal rotation streching technique for overhand and throwing athletes. J Orthop Sports Phys Ther 21:216-219

12. Altman DG (1991) Practical statistics for medical research. Chapman \& Hall, London

13. Burkhart SS, Morgan CD, Kibler WB (2003) The disabled throwing shoulder: spectrum of pathology. Part I: pathoanatomy and biomechanics. Arthroscopy 19:404-420

14. Cools AM, Witvrouw E, Declercq G, Vanderstraeten G, Cambier D (2004) Evaluation of isokinetic force production and associated muscle activity in the scapular rotators during a protractionretraction movement in overhead athletes with impingement symptoms. Br J Sports Med 38:64-68

15. Cools AM, Dewitte V, Lanszweert F, Notebaert D, Roets A, Soetens B et al (2007) Rehabilitation of scapular muscle balance: which exercises to prescribe? Am J Sports Med 35:1744-1751

16. Smith J, Dietrich CT, Kotajarvi BR, Kaufman KR (2006) The effect of scapular protraction on isometric shoulder rotation strength in normal subjects. J Shoulder Elbow Surg 15:339343 
17. Nakamizo H, Nakamura Y, Nobuhara K, Yamamoto T (2008) Loss of glenohumeral internal rotation in little league pitchers: a biomechanical study. J Should Elbow Surg 17:795-801

18. Happee R, van-der-Helm FC (1995) The control of shoulder muscles during goal directed movements, an inverse dynamic analysis. J Biomech 28:1179-1191

19. Kibler WB, Sciascia A (2009) Scapular dyskinesis: current concepts. Br J Sports Med. doi:10.1136/bjsm.2009.058834

20. McClure PM, Michener LA, Sennett BJ, Karduna AR (2001) Direct 3-dimensional measurement of scapular kinematics during dynamic movements in vivo. J Shoulder Elbow Surg 10:269-277

21. Rokito As, Jobe FW, Pink MM, Perry J, Brault J (1998) Electromyogrphic analysis of shoulder function durong the volleyball serve and spike. J Shoulder Elbow Surg 7:256-263
22. David G, Magarey ME, Jones MA, Dvir Z, Turker KS, Sharpe M (2000) EMG and strength correlates of selected shoulder muscles during rotations of the glenohumeral joint. Clin Biomech (Bristol, Avon) 15:95-102

23. Escamilla RF, Andrews JR (2009) Shoulder muscle recruitment patterns and related biomechanics during upper extremity sports. Sports Med 39:569-590

24. Escamilla RF, Yamashiro K, Paulos L, Andrews JR (2009) Shoulder muscle activity and function in common shoulder rehabilitation exercises. Sports Med 39:663-685 